\title{
La combinación de trimetoprima-Sulfametoxazol (Cotrimoxazol) se asocia a muerte súbita en ancianos que toman espironolactona
}

Trimethoprim-Sulfamethoxazole (Cotrimoxazole) is associated with sudden death in the elderly taking spironolactone

CMAJ. 2015;187(4):E138-43

\section{Objetivo}

La trimetoprima-sulfametoxazol (TMS) aumenta el riesgo de hiperpotasemia cuando se usa con espironolactona. Se postula que esta combinación podría potenciar arritmias fatales y muerte súbita.

\section{Objetivos}

Evaluar el riesgo de muerte súbita asociado a la combinación de espironolactona y TMS.

\section{Diseño, lugar, participantes}

Estudio de casos y controles anidado en una población de adultos mayores de 66 años de la ciudad de Ontario que habían recibido espironolactona entre 1994 y 2011. Dentro de este grupo fueron identificados como casos los pacientes que fallecieron de muerte súbita luego de los 14 días de recibir TMS $\mathrm{u}$ otros antibióticos (amoxicilina, ciprofloxacina, norfloxacina o nitrofurantoína), excluyendo a los inmunosuprimidos.

Para cada caso fueron identificados cuatro controles apareados por edad y sexo, determinándose el odds ratio (OR) para la asociación entre muerte súbita y exposición a cada antibióti$\mathrm{co}$, en relación a la amoxicilina (usado como comparador), ajustando por predictores de muerte súbita y utilizando un índice de riesgo de enfermedad.

\section{Resultados principales}

De los 11.968 pacientes que sufrieron muerte súbita mientras que recibían espironolactona, fueron identificados 328 cuyas muertes ocurrieron dentro de los 14 días después de la exposición al antibiótico. Los pacientes tenían una mediana de edad de 86 años (rango intercuartílico de 81 a 90).

Comparado con amoxicilina, TMS estuvo asociada con más del doble de riesgo de muerte súbita (OR ajustado 2,46, IC95\% 1,55 a 3,90). Ciprofloxacina (1,55, IC95\% 1,02 a 2,38) y nitrofurantoína $(1,70$, IC $95 \% 1,03$ a 2,79) también se asociaron con un riesgo aumentado de muerte súbita, aunque los riesgos de la nitrofurantoína no se sostuvieron en el análisis de sensibilidad.

\section{Conclusiones}

El antibiótico TMS se asoció con un riesgo aumentado de muerte súbita entre los pacientes adultos que tomaban espironolactona. Cuando se lo considere clínicamente apropiado, se deben considerar regímenes antibióticos alternativos en esta subpoblacíón.

Fuente de financiamiento: Canadian Drug Safety and Effectiveness Research Network. Institute for Clinical Evaluative Sciences.

\section{Comentario}

El estudio presenta limitaciones inherentes a su diseño metodológico: potencial sesgo por clasificación inadecuada, tanto de casos como de controles (uso retrospectivo de bases de datos administrativas) y la presencia de factores confundidores no controlados (p. ej. el tipo de enfermedad que hizo que requiriera antibioticoterapia y el estado general del paciente al recibir antibiótico). Estos disminuyen nuestra confianza en la fortaleza de la asociación identificada.

El antibiótico TMS aumenta la concentración plasmática de potasio mediante la inhibición de la excreción de la nefrona distal de manera similar al diurético ahorrador de potasio amilorida. Este efecto se suma al antagonismo de la aldosterona producido por la espironolactona. Algunos factores de riesgo identificados con la hiperpotasemia inducida por TMS son: dosis altas del antibiótico, insuficiencia renal crónica, edad avanzada, hipoaldosteronismo (incluido el inducido por drogas) e interacción con otros fármacos que inducen elevación de la potasemia (antiinflamatorios no esteroideos y diuréticos ahorradores de potasio)'.

Actualmente, y dado que existe mayor resistencia para las enterobacterias causantes de infección urinaria ${ }^{2}$, las indicaciones de TMS fueron cambiando. Sin embargo, el uso de TMS cada vez está más difundido para las infecciones de partes blandas con sospecha de Staphylococcus aureus meticilinoresistente de la comunidad ${ }^{3}$ (SAMR-C).

\section{Conclusiones del comentador}

Si bien este estudio no muestra una fuerte causalidad directa entre la muerte súbita y la combinación de antibióticos, es un llamado a la atención para reflexionar sobre determinadas asociaciones farmacológicas no deseables y para repensar las estrategias disponibles para la minimización de riesgos en estas poblaciones vulnerables.

Juan Víctor Ariel Franco [ Servicio de Medicina Familiar y Comunitaria del Hospital Italiano de Buenos Aires. Departamento de Toxicología y Farmacología de la Facultad de Medicina de la Universidad de Buenos Aires. Centro Cochrane del Instituto Universitario del Hospital Italiano de Buenos Aires juan.franco@ hiba.org.ar]

Franco J. La combinación de trimetoprima-Sulfametoxazol (Cotrimoxazol) se asocia a muerte súbita en ancianos que toman espironolactona. Evid Act Pract Ambul. $2016 ; 19(3) 91$. Comentado de: Antoniou T, y col. Trimethoprim-sulfamethoxazole and risk of sudden death among patients taking spironolactone. CMAJ. 2015;187(4):E138-43. PMID: 25646289.

Referencias

1. Perazella M. Trimethoprim-induced hyperkalaemia: clinical data, mechanism, prevention and management. Drug Saf. 2000 Mar;22(3):227-36.

2. Bryce A. Global prevalence of antibiotic resistance in paediatric urinary tract infections caused by Escherichia coli and association with routine use of antibiotics in primary care: systematic review and meta-analysis. BMJ. 2016 Mar 15;352:i939. doi: 10.1136/bmj.i939.

3. Miller L. DMID 07-0051 Team. Clindamycin versus trimethoprim-sulfamethoxazole for uncomplicated skin infections. N Engl J Med. 2015 Mar 19;372(12):1093-103. doi: 10.1056/NEJMoa1403789. 